\title{
Increased production of suilysin contributes to invasive infection of the Streptococcus suis strain $05 \mathrm{ZYH33}$
}

\author{
ZHENGXIN HE ${ }^{1,2^{*}}$, YAYA PIAN ${ }^{2 *}$, ZHIQIANG REN $^{2}$, LILI BI $^{2}$, YUAN YUAN $^{2}$, \\ YULING ZHENG $^{2}$, YONGQIANG JIANG ${ }^{2}$ and FUKUN WANG ${ }^{1}$ \\ ${ }^{1}$ Department of Clinical Laboratory, Bethune International Peace Hospital of PLA, Shijiazhuang, Hebei 050082; \\ ${ }^{2}$ State Key Laboratory of Pathogen and Biosecurity, Institute of Microbiology and Epidemiology, \\ Academy of Military Medical Sciences, Beijing 100071, P.R. China
}

Received December 1, 2013; Accepted June 5, 2014

DOI: $10.3892 / \mathrm{mmr} .2014 .2586$

\begin{abstract}
Streptococcus suis serotype 2 (SS2) is widely recognized in the veterinary world as the cause of rapidly progressive and fatal sepsis in infant pigs, manifested with meningitis, polyarthritis and pneumonia. It has evolved into a highly infectious strain, and caused two large-scale outbreaks of human epidemic in China, characterized bytypical toxic-shock syndrome and invasive infection. However, the molecular basis of virulence of this emerging zoonotic pathogen is still largely unknown. The present study shows that the sequence type (ST)7 epidemic strain S. suis 05ZYH33 causes higher mortality, higher necrosis of polymorphonuclear neutrophils and a significantly higher damage to human umbilical vein endothelial cells compared to the non-epidemic strain S. suis 1940. These differences appear to associate with the enhanced secretion of suilysin (sly) by $S$. suis $05 Z$ YH33 compared to the non-epidemic strain 1940. Inclusion of additional strains confirmed that the epidemic ST7 strains produce more sly protein (mean, $1.49 \mathrm{~g} / \mathrm{ml}$; range, 0.76-1.91 g/ml) than non-epidemic strains (mean, $0.33 \mathrm{~g} / \mathrm{ml}$; range, $0.07-0.94 \mathrm{~g} / \mathrm{ml}$ ), and this difference is significant $(\mathrm{P}<0.001)$. The nonpolar mutant strain $S$. suis $\Delta s l y$, constructed from the epidemic ST7 strain S. suis 05ZYH33 confirmed the role of sly on the enhanced virulence
\end{abstract}

Correspondence to: Professor Yongqiang Jiang, State Key Laboratory of Pathogen and Biosecurity, Institute of Microbiology and Epidemiology, Academy of Military Medical Sciences, 20 Dongdajie Street, Beijing 100071, P.R. China

E-mail: jiang_yongqiang@sina.cn

Professor Fukun Wang, Department of Clinical Laboratory, Bethune International Peace Hospital of PLA, 398 Zhongshan Road, Shijiazhuang, Hebei 050082, P.R. China

E-mail:wangfk8@sina.com

*Contributed equally

Key words: suilysin, invasive infection, epidemic strain, Streptococcus suis of S. suis ST7 strains. These findings suggest that increased sly production in $S$. suis $05 Z$ YH33 facilitates penetration to the epithelium and its survival in the bloodstream, thereby contributing to the invasive infection.

\section{Introduction}

Streptococcus suis is an important emerging human threat that can cause severe systemic infection (1-3). Since the first reported cases of human infection by $S$. suis in 1968, the number of cases has significantly increased in the past few years, reaching $\sim 1,000$ cases by now, with the majority occurring in Southeast Asia. S. suis infections are the third most common cause of community-acquired bacterial meningitis in Hong Kong, and the leading cause of adult meningitis in Vietnam (4-5).

The $S$. suis serotype 2 (SS2) is the most common cause of the disease in humans, although serotypes 1, 4, 14 and 16 have also been reported to cause severe disease in a limited number of individuals. Most previous studies have concerned sporadic cases of $S$. suis infection, but two Chinese outbreaks (in 1998 and 2005) that involved $>200$ cases and 50 deaths, emphasized the importance of $S$. suis as an emerging zoonotic pathogen $(6,7)$. The most important feature of these outbreaks was the high incidence of systemic disease, the proportionally few cases of meningitis, and the high mortality. Epidemiological surveys have indicated that all patients had a history of close contact with diseased pigs and pork-derived products, and that the emergence of the highly virulent SS2 strains played a key role in the severe outbreaks $(8,9)$.

The current understanding of the pathogenesis of $S$. suis remains limited. Whole-genome sequencing and comparative genomic analysis (10) revealed that a DNA fragment of $\sim 89 \mathrm{~kb}$, designated as $89 \mathrm{~K}$, is present in the two Chinese strains (98HAH12 and 05ZYH33), and absent in the P1/7, which is a widely epidemic virulent Streptococcus suis serotype 2 strain. The $89 \mathrm{~K}$ fragment was proposed to be a pathogenicity island, and genetic studies indicated that disruption of the Salk/SalR two-component system (TCS) inside the $89 \mathrm{~K}$ considerably attenuates the virulence of the pathogen, whereas functional complementation restores virulence in infection experiments of piglets (11). A recent study (12) showed that 
$89 \mathrm{~K}$ can spontaneously excise to form an extrachromosomal circular product, and the $89 \mathrm{~K}$ excision intermediate can act as a substrate for lateral transfer to non-89K SS2 recipients via a genomic island type IV secretion system (T4SS) encoded in $89 \mathrm{~K}$. The authors proposed that these genetic events are important for the emergence, pathogenesis and persistence of epidemic SS2 strains. Based on sequence typing (ST), it was also suggested that ST7 strains are prevalent in China and possess a stronger capacity to stimulate $\mathrm{T}$ cells, naive $\mathrm{T}$ cells and peripheral blood mononuclear cell proliferation compared to ST1 strains (13-15). The authors of these studies proposed a two-stage hypothesis to explain streptococcal toxic shock syndrome in the Chinese outbreaks. However, both hypotheses need to be further investigated in the future.

Pore-forming toxins (PFTs) are the most prevalent virulence factors produced by disease-causing bacteria, and are required for the virulence of numerous important human pathogens, including Staphylococcus aureus, S. pyogenes, Clostridium perfringens and Aeromonas hydrophilia (16). A PFT produced by $S$. suis known as suilysin (sly) has been recognized as a virulence factor owing to its toxicity to the host epithelial cells, endothelial cells and macrophages (17-19). The sly protein belongs to a family of thiol-activated toxins and has a molecular weight of $54 \mathrm{kDa}$. It is a cholesterol-dependent cytolysin related to the streptolysin $\mathrm{O}$ of $S$. pyogenes and to pneumolysin of $S$. pneumoniae. Immunization to sly provides protection against lethal challenge with a serotype 2 strain in both mice and pig modelss $(20,21)$. However, the expression profile of sly in certain $S$. suis populations and its role in causing invasive infections have not been characterized. In this study, we found that increased production of sly in the S. suis strain $05 Z$ YH33 is associated with enhanced severity of $S$. suis infections and thus, may contribute to the translocation of the pathogen across the epithelial barrier. These results indicate that enhanced sly production may play an important role during $S$. suis invasive infections.

\section{Materials and methods}

Ethics. CD1 mice were obtained from the Experimental Animal Centre of the Academy of Military Medical Sciences (Beijing, China). Animal welfare and experimental procedures were approved by the Academy of Military Medical Sciences Animal Care and Use Committee, and were conducted in strict accordance with the Guide for the Care and Use of Laboratory Animals (National Research Council of USA, 1996). Efforts were made to minimize animal suffering and to reduce the number of animals used. For the survival experiments, the decisions were made following the relevant guidelines of the Organisation for Economic Co-operation and Development [OECD Environmental Health and Safety Publications, Series on Testing and Assessment no. 19, ENV/JM/MONO(2000)7].

Bacterial strains and growth conditions. The bacterial strains used in this study are listed in Table I. S. suis was grown in Difco $^{\text {TM }}$ Todd-Hewitt broth (THB; Becton, Dickinson and Company, Franklin Lakes, NJ, USA) at $37^{\circ} \mathrm{C}$. For antibiotic selection, $5 \mu \mathrm{g} / \mathrm{ml}$ of chloramphenicol $(\mathrm{Cm})$ or $100 \mu \mathrm{g} / \mathrm{ml}$ spectinomycin $(\mathrm{Spc})$ were used.
Cell culture and lactate dehydrogenase $(\mathrm{LDH})$ release assay. Human umbilical vein endothelial cells (HUVECs; Institute of Biochemistry and Cell Biology, Shanghai, China) were cultured in RPMI-1640 medium containing 10\% fetal bovine serum (FBS). Cells $(200 \mu \mathrm{l})$ were added at a density of $5 \times 10^{4}$ cells $/ \mathrm{ml}$ into 96 -well culture plates. S. suis strains $05 Z$ YH33 or 1940 at the mid-log phase of growth were resuspended in cell culture medium and were added to the cell culture wells at multiplicity of infection (MOI) of 100. The amount of released lactate dehydrogenase (LDH) from HUVECs was calculated with an LDH release assay kit (Promega, Madison, WI, USA) after $4 \mathrm{~h}$ of incubation.

Targeted mutagenesis of the sly gene. Polymerase chain reaction (PCR) was used to generate an in-frame substitution of the sly gene with the $\mathrm{Cm}$ gene using a previously described method (22). Briefly, 644 and 590 bp immediately upstream and downstream of the sly coding sequence (SSU05_1403) were amplified with the primer pairs sly_upF/_upR and $s l y \_$downF/_downR, respectively. The $C m$ gene was amplified using the primer pair Cm_F/_R (Table II). The PCR products were cloned into the Invitrogen ${ }^{\mathrm{TM}}$ pCR2.1 plasmid (Thermo Fisher Scientific, Waltham, MA, USA), digested using restriction enzymes and subcloned into the plamsid pSET4s one by one. Allelic exchange mutagenesis in S. suis 05ZYH33 was performed as previously described (22) to generate the mutant $\Delta s l y$. Allelic replacement of sly with $\mathrm{Cm}$ in the $\mathrm{S}$. suis chromosome was confirmed by PCR and sequence analysis using the Phusion High-Fidelity DNA Polymerase reagents (New England Biolabs, Ipswich, MA, USA) on a ABI 3730XL machine (Applied Biosystems, Carlsbad, CA, USA).

Enzyme-linked immunosorbent assay (ELISA) assay. The sly level in the supernatant of different $S$. suis strain cultures was determined using a double antibody sandwich ELISA assay. The antibodies used were polyclonal. Briefly, purified rabbit anti-sly IgG (1:5,000 dilution) was used to coat the wells of a microtiter plate. Purified rat anti-sly $\operatorname{IgG}(1: 5,000)$ was used as the primary antibody, peroxidase-conjugated goat antirat antibody $(1: 5,000)$ was used as the secondary antibody, and K-Blue was used as the substrate (all from Santa Cruz Biotechnology, Santa Cruz, CA, USA). After 10 min of incubation at room temperature, the reaction was terminated with addition of $2 \mathrm{~N} \mathrm{H}_{2} \mathrm{SO}_{4}$, and the optical density was measured at $450 \mathrm{~nm}\left(\mathrm{OD}_{450}\right)$ using the SpectraMax ${ }^{\circledR}$ Plus384 Absorbance Microplate reader (Molecular Devices LLC, Sunnyvale, CA, USA). Purified sly protein (23) was used to establish the standard curve.

Hemolytic activity assay. The haemolytic activity assay was performed using a previously described method (24). Briefly, the culture supernatant of $S$. suis strains was diluted 2-fold in phosphate-buffered saline (PBS). An equal amount of human red blood cells (RBCs), obtained from the Department of Hematology (Hospital 307 of Chinese People's Liberation Army, Beijing China) and washed twice in PBS, was added to $0.5 \mathrm{ml}$ of each dilution (final concentration of RBCs, 1.4\%). Following incubation for $1 \mathrm{~h}$ at $37^{\circ} \mathrm{C}$, the mixtures were sedimented by centrifugation $(1,500 \mathrm{x}$ g for $10 \mathrm{~min})$, and the supernatants were transferred to microplates. Following correction with the 
Table I. Bacterial strains and plasmids used in this study.

\begin{tabular}{|c|c|c|c|c|}
\hline Material & Description & Area (year)-Origin & Status & Source/Ref. \\
\hline \multicolumn{5}{|c|}{ Streptococcus suis strains } \\
\hline$\Delta s l y$ & $\begin{array}{l}\text { In frame deletion of sly } \\
\text { in } 05 \mathrm{ZYH} 33\end{array}$ & Beijing, China (2011) - Our lab & Mutant & This study \\
\hline 1330 & Sly-89K- & Canada-HPL & Avirulent & $(25)$ \\
\hline 05ZYH33 & $\mathrm{Sly}^{+} 89 \mathrm{~K}^{+}$ & Sichuan, China (2005)-HP & Epidemic & This study \\
\hline 98001 & $\mathrm{Sly}^{+} 89 \mathrm{~K}^{+}$ & Jiangsu, China (1998)-HP & Epidemic & This study \\
\hline 99001 & $\mathrm{Sly}^{+} 89 \mathrm{~K}^{+}$ & Jiangsu, China (1998)-HP & Epidemic & This study \\
\hline 98003 & $\mathrm{Sly}^{+} 89 \mathrm{~K}^{+}$ & Jiangsu, China (1998)-HP & Epidemic & This study \\
\hline 98005 & $\mathrm{Sly}^{+} 89 \mathrm{~K}^{+}$ & Jiangsu, China (1998)-HP & Epidemic & This study \\
\hline 98012 & $\mathrm{Sly}^{+} 89 \mathrm{~K}^{+}$ & Jiangsu, China (1998)-HP & Epidemic & This study \\
\hline 98015 & $\mathrm{Sly}^{+} 89 \mathrm{~K}^{+}$ & Jiangsu, China (1998)-HP & Epidemic & This study \\
\hline 98242 & $\mathrm{Sly}^{+} 89 \mathrm{~K}^{+}$ & Jiangsu, China (1998)-HP & Epidemic & This study \\
\hline 4 & $\mathrm{Sly}^{+} 89 \mathrm{~K}^{+}$ & Sichuan, China (2005)-DP & Epidemic & This study \\
\hline 5 & $\mathrm{Sly}^{+} 89 \mathrm{~K}^{+}$ & Sichuan, China (2005)-DP & Epidemic & This study \\
\hline SUN & $\mathrm{Sly}^{+} 89 \mathrm{~K}^{+}$ & Jiangsu, China (2006)-HP & Epidemic & This study \\
\hline 606 & $\mathrm{Sly}^{+} 89 \mathrm{~K}^{-}$ & China (1980)-DP & Non-epidemic & This study \\
\hline 607 & $\mathrm{Sly}^{+} 89 \mathrm{~K}^{-}$ & Japan-DP & Non-epidemic & This study \\
\hline 1940 & $\mathrm{Sly}^{+} 89 \mathrm{~K}^{-}$ & China (1980)-DP & Non-epidemic & This study \\
\hline 1941 & $\mathrm{Sly}^{+} 89 \mathrm{~K}^{-}$ & China (1980)-DP & Non-epidemic & This study \\
\hline NJ & $\mathrm{Sly}^{+} 89 \mathrm{~K}^{-}$ & Jiangsu, China-DP & Non-epidemic & This study \\
\hline 4005 & $\mathrm{Sly}^{+} 89 \mathrm{~K}^{-}$ & The Netherlands-DP & Non-epidemic & (26) \\
\hline s735 & $\mathrm{Sly}^{+} 89 \mathrm{~K}^{-}$ & The Netherlands-DP & Non-epidemic & (27) \\
\hline T15 & $\mathrm{Sly}^{+} 89 \mathrm{~K}^{-}$ & Europe-HPL & Non-epidemic & $(28)$ \\
\hline \multicolumn{5}{|l|}{ Plasmids } \\
\hline pCR2.1 & na & na & na & Invitrogen $^{\mathrm{TM}}$ \\
\hline pSET4s & na & na & na & (29) \\
\hline
\end{tabular}

Sly, suilysin; 89K, 89 kb pathogenicity island; HPL, healthy piglets; HP, human patients; DP, diseased piglets; na, not applicable.

controls, which lacked either haemolysin or erythrocytes, the $\mathrm{OD}_{540}$ of the supernatant of each dilution was measured on a SpectraMax Plus Absorbance Microplate reader.

Reverse transcription-quantitative-PCR (RT-qPCR) analysis The RT-qPCR analysis was performed with a sly gene-specific primer set (forward, ACTTACGAGCCACAAGAGATTC and reverse, GCAGCCTTAGCATCAATAACAG) on cDNA from the $S$. suis strains 05ZYH33 and 1940. The assays were carried out in triplicate using reagents of the SYBR Premix Ex Taq ${ }^{\mathrm{TM}}$ mastermix (Takara Bio, Co., Ltd., Shiga, Japan) on an Opticon 2 system (MJ Research, Waltham, MA, USA) using RNA isolated from three independent cultures for each strain. RNA was extracted using the TRIzol RNA Reagent (Invitrogen Life Technologies, Carlsbad, CA, USA) following the manufacturer's instructions. The PCR cycling conditions were as follows: $95^{\circ} \mathrm{C}$ for $10 \mathrm{~min}$ followed by 40 cycles of $95^{\circ} \mathrm{C}$ for $15 \mathrm{sec}$ and $60^{\circ} \mathrm{C}$ for $1 \mathrm{~min}$. The gene encoding the $16 \mathrm{~S}$ rRNA was used as an internal control. The $\mathrm{Ct}$ values were normalized to the average $\mathrm{Ct}$ (30) and the mean fold-changes for the target genes were calculated as described by Livak and Schmittgen (31).
Polymorphonuclear neutrophil (PMN) phagocytosis assay. The PMN phagocytosis assay was performed as previously described (32). Human PMNs were isolated from healthy human blood as follows: blood was incubated for $40 \mathrm{~min}$ at room temperature in a ratio of 3:1 ratio of $6.0 \%$ Dextran to sediment erythrocytes. Ficoll, $70 \%$ percoll and blood supernatant were combined (1:1:1) and centrifuged at $400 \mathrm{x} \mathrm{g}$ for $30 \mathrm{~min}$ at room temperature to separate PMNs. The PMNs were aspirated in to a new cuvette, resuspended in 1640-RPMI, and enumerated by microscopy. Briefly, $1 \mathrm{ml}$ of human PMNs $\left(10^{7}\right.$ cells) were mixed with an equal number of pre-opsonized S. suis colonies and incubated for $15 \mathrm{~min}$ at $37^{\circ} \mathrm{C}$ under continuous rotation to allow phagocytosis. When needed, different concentrations of recombinant sly protein (23) were added (50-500 ng). To quantify the intracellular bacteria, extracellular $S$. suis were killed by incubating with $100 \mu \mathrm{g} / \mathrm{ml}$ gentamicin and $5 \mathrm{~g} / \mathrm{ml}$ penicillin for $1 \mathrm{~h}$ at $4^{\circ} \mathrm{C}$. Subsequently, the cells were washed twice with PBS and lysed for $15 \mathrm{~min}$ at room temperature in $400 \mu \mathrm{l}$ of $1 \%$ saponin. The viable bacteria were counted on an inverted IX81 microscope (Olympus, Tokyo, Japan) after plating serial dilutions of the supernatants on THB agar plates. 
Table II. Oligonucleotide primer sequences used in this study.

\begin{tabular}{llc}
\hline Primer & \multicolumn{1}{c}{ Sequence (5'-3') } & Restriction enzyme \\
\hline Sly_upF & GGGGG_AAGCTTCTAGTCGGGGGAGTTTTTGTG & HindIII \\
Sly_upR & GGGGGGGTCGACTTAATATCTTGTTTGGAATCTG & SalI \\
Cm_F & GCGGTCGACTAATTCGATGGGTTCCGAGG & SalI \\
Cm_R & CGCGGATCCCACCGAACTAGAGCTTGATG & BamHI \\
Sly_downF & GGGGGGATCCCATGGGAGTGGTGGAGAACAGT & BamHI \\
Sly_downR & GGGGGGGGAATTCTTGGCCCGAATACCGACAG & EcoRI \\
\hline
\end{tabular}

Sly, suilysin; up, upstream; down, downstream; F, forward; R, reverse; $\mathrm{Cm}$, chloramphenicol; underlined, restriction sites.

Mouse infections. CD1 female mice (6-week-old) were chosen to evaluate the virulence of the S. suis strains 05ZYH33 and 1940. Briefly, mice ( $\mathrm{n}=10$ per group) were intraperitoneally injected with $10^{8}$ living colony forming units (CFUs) of $S$. suis strains 05ZYH33 and 1940 in $0.1 \mathrm{ml}$ sterile saline. The mortality of mice infected with these bacterial strains was recorded at 4-h intervals for $32 \mathrm{~h}$. To count the bacteria present in the blood, blood samples were collected from the tail vein and plated onto THB agar to accurately determine the viable bacteria at $5 \mathrm{~h}$ post-infection. To assess the necrosis of PMNs, we sacrificed the mice and injected $5 \mathrm{ml}$ of RPMI-1640 medium containing $10 \%$ FBS into the abdominal cavities. Then, mice were surgically opened and 4-ml exudates were collected with $23-\mathrm{G}$ needles. The exudates were centrifuged at $500 \mathrm{x}$ g for $5 \mathrm{~min}$, and cell pellets were resuspended in $100 \mathrm{ml}$ of staining buffer (PBS containing 1\% goat serum). The samples were stained either with fluorescein isothiocyanate (FITC)-conjugated antimouse anti-Ly-6G (a neutrophil marker), or with the appropriate isotype control antibody (eB149/10H5; both from eBiosciences, San Diego, CA, USA). Propidium iodide $(0.5 \mathrm{mg} / \mathrm{ml}$; BD Biosciences, San Jose, CA, USA) was used to determine the cell viability. Samples were analyzed on a FACSCalibur flow cytometer and the necrotic PMNs were quantified with the CellQuest software (both from BD Biosciences). Data were obtained from 30,000 cells for each sample.

CD1 female mice (6-week-old, 22 g) were chosen to evaluate the virulence of the $S$. suis strain 05ZYH33 and the $\Delta$ sly mutant. Mice ( $n=10$, per group) were intraperitoneally injected with $0.5 \mathrm{ml}\left(\sim 5 \times 10^{8} \mathrm{CFUs}\right)$ of $S$. suis at the late-exponential growth phase in THB medium. The mortality of the infected mice was recorded for $50 \mathrm{~h}$, and the bacteria in the blood were counted at different time-points post-infection. To histopathologically assess the severity of changes in the abdominal wall tissues, mice were surgically opened $6 \mathrm{~h}$ after injection. The abdominal wall tissues were fixed in $10 \%$ Formaldehyde (Sigma, St. Louis, MO, USA), sectioned and stained with hematoxylin and eosin. The slides were observed and photographed using a BX53 fluorescence microscope (Olympus, Tokyo, Japan) equipped with a DP72 CCD camera.

Statistical analysis. Statistical analysis was performed using the SPSS 13.0 version software (SPSS Inc., Chicago, IL, USA). Statistical significance of differences was assessed with Student's t-tests, with a p-value $<0.05$ considered to indicate statistically significant differences.

\section{Results}

The S. suis epidemic strain is more virulent than the non-epidemic strain. The $S$. suis epidemic strains are expected to show high virulence, and need to resist host defence mechanisms and be able to invade the epithelial or the endothelial barrier in order to cause successful infection. To evaluate the potential differences in virulence between $S$. suis epidemic and non-epidemic strains, CD1 female mice were intraperitoneally injected with $10^{8}$ CFUs of the $S$. suis strains 05ZYH33 (epidemic, ST7) or 1940 (non-epidemic) in $0.1 \mathrm{ml}$ sterile saline. The strain 05ZYH33 caused significantly higher mortality $(\mathrm{p}<0.05)$ than the 1940 strain (Fig. 1A). The bacterial concentration in the blood of the infected mice also appeared different at $5 \mathrm{~h}$ post-infection, although the difference was not statistically significant (Fig. 1B). In addition, S. suis 05ZYH33 caused higher necrosis of PMNs (Fig. 1C), the major cell type mediating acute inflammatory responses to bacterial infections, compared to $S$. suis $1940(13.21 \pm 3.11$ vs. $9.08 \pm 2.56 \%)$. Next, we used HUVECs to test the ability of the two $S$. suis strains to damage the cells. The results showed that 05ZYH33 has a higher cytotoxicity than 1940, as determined by the LDH assay (Fig. 1D). Taken together, these data demonstrate that the $S$. suis epidemic strain 05ZYH33 is more virulent than the non-epidemic strain 1940.

Next, we investigated whether the sly protein may contribute to the enhanced virulence of the $S$. suis epidemic strain. Pre-opsonised S. suis 05ZYH33 or 1940 were incubated with purified human PMNs (MOI =1) for $15 \mathrm{~min}$ in the presence or absence of the sly protein, and the bacteria phagocytized by PMNs were counted. This assay showed that PMNs phagocytized the $S$. suis 1940 strain at higher rates compared to the 05ZYH33 strain, (Fig. 1E) while adding sly protein significantly decreased the phagocytosis of S. suis 1940 by the PMNs.

The S. suis epidemic strain 05ZYH33 shows increased production of sly compared to the non-epidemic strains. Since the sly protein displays hemolytic activity, we then compared the relative amounts of sly produced by the $S$. suis epidemic strain 05ZYH33 and the non-epidemic strain 1940. Although both strains showed the same growth rate (Fig. 2A), the supernatant of $S$. suis 05ZYH33 displayed higher hemolytic activity than that of S. suis 1940 (Fig. 2B). This phenotypic difference was further confirmed by qRT-PCR, which indicated a difference 

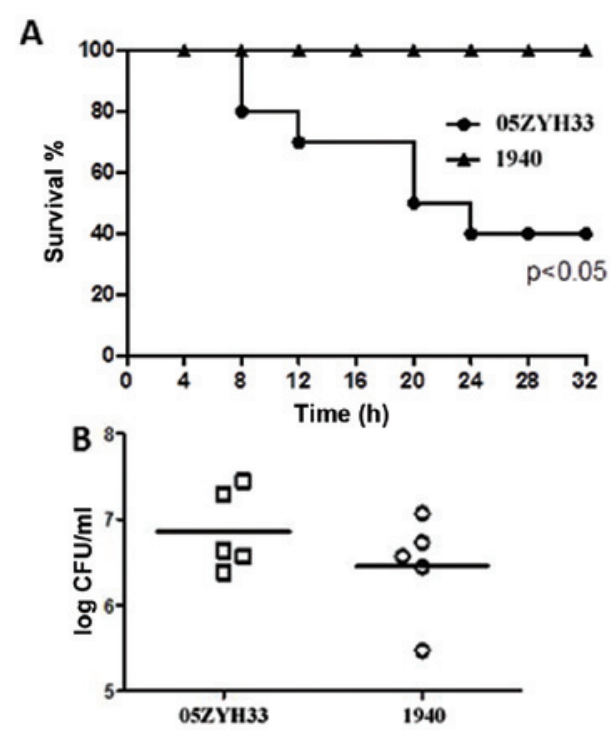

C
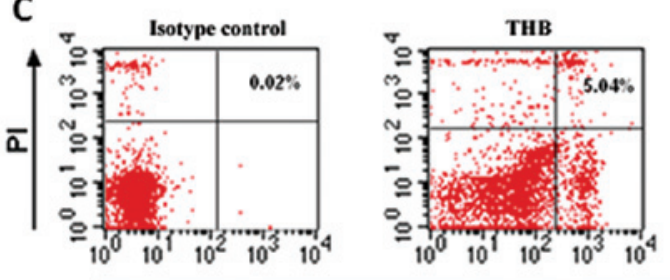

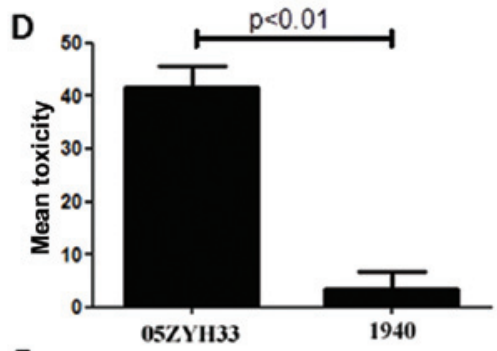

E
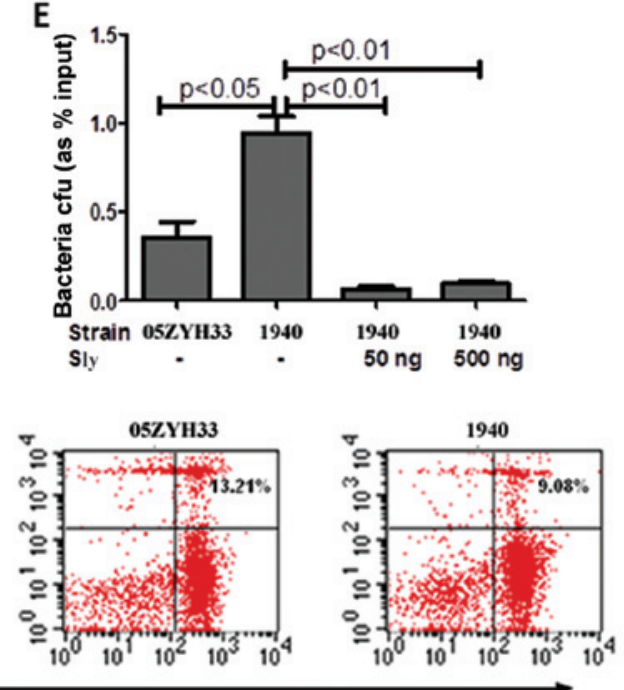

Ly-6G-FITC

Figure 1. Streptococcus suis epidemic strain 05ZYH33 is more virulent than the non-epidemic strain 1940. (A) CD1 female mice were intraperitoneally injected with $10^{8} \mathrm{CFUs}$ of living S. suis $05 \mathrm{ZYH} 33$ and $1940 \mathrm{in} 0.1 \mathrm{ml}$ sterile saline. The mortality of mice was recorded at 4 -h intervals for $32 \mathrm{~h}$. Cumulative results from three independent experiments, each with 10 animals per group, are shown. (B) Bacteria in the blood ( $\mathrm{n}=5$, per group) were counted at $5 \mathrm{~h}$ post-infection. (C) The necrosis of PMNs in mice infected by different bacterial strains was assayed using fluorescein isothiocyanate (FITC)-conjugated anti-mouse anti-Ly-6G and propidium iodide (PI) staining. Data represent the values obtained in four independent experiments. THB, Todd-Hewitt broth. (D) S. suis $05 \mathrm{ZYH} 33$ or $1940\left(10^{8} \mathrm{CFUs}\right)$ were incubated with human umbilical vein endothelial cells $(\mathrm{MOI}=100)$ for 4 h. Cytotoxicity was determined by the lactate dehydrogenase release assay. The data represent the mean \pm standard deviation from three independent experiments. (E) Pre-opsonised $S$. suis 05ZYH33 or $1940\left(10^{7} \mathrm{CFUs}\right)$ were incubated with purified human polymorphonuclear neutrophils $(\mathrm{MOI}=1)$ in the presence or absence of recombinant sly protein for $15 \mathrm{~min}$. The number of bacteria phagocytized by the PMNs was counted.

A

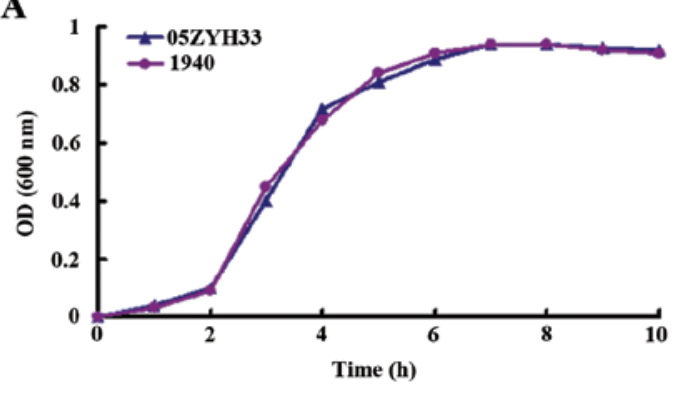

B

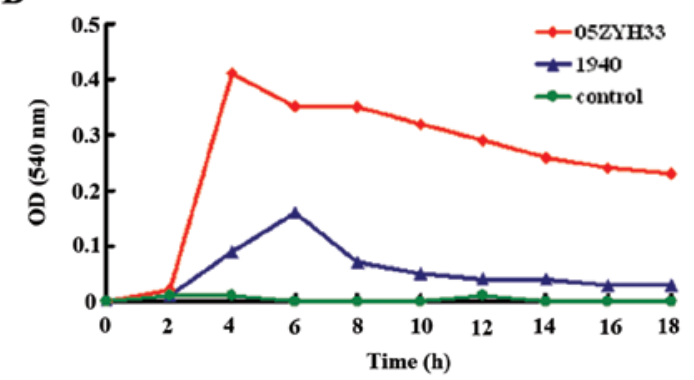

C

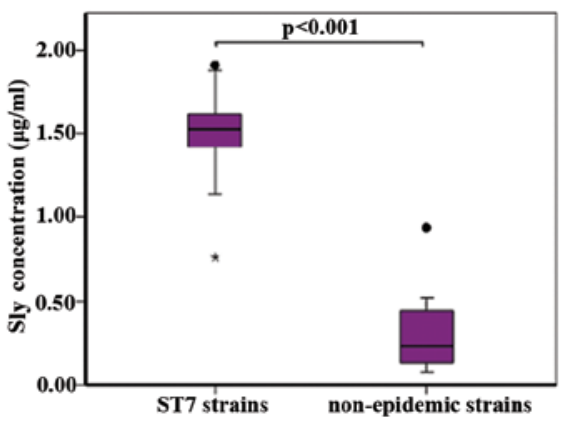

Figure 2. Streptococcus suis ST7 epidemic strains show increased production of suilysin (sly) compared to non-epidemic strains. S. suis 05ZYH33 and 1940 (A) growth curves and (B) relative hemolytic activities, assessed in the supernatants collected at different time-points. (C) Sly protein concentration, determined by an enzyme-linked immunosorbent assay, performed on 11 ST7 strains and 8 non-epidemic strains listed in Table I. 
B
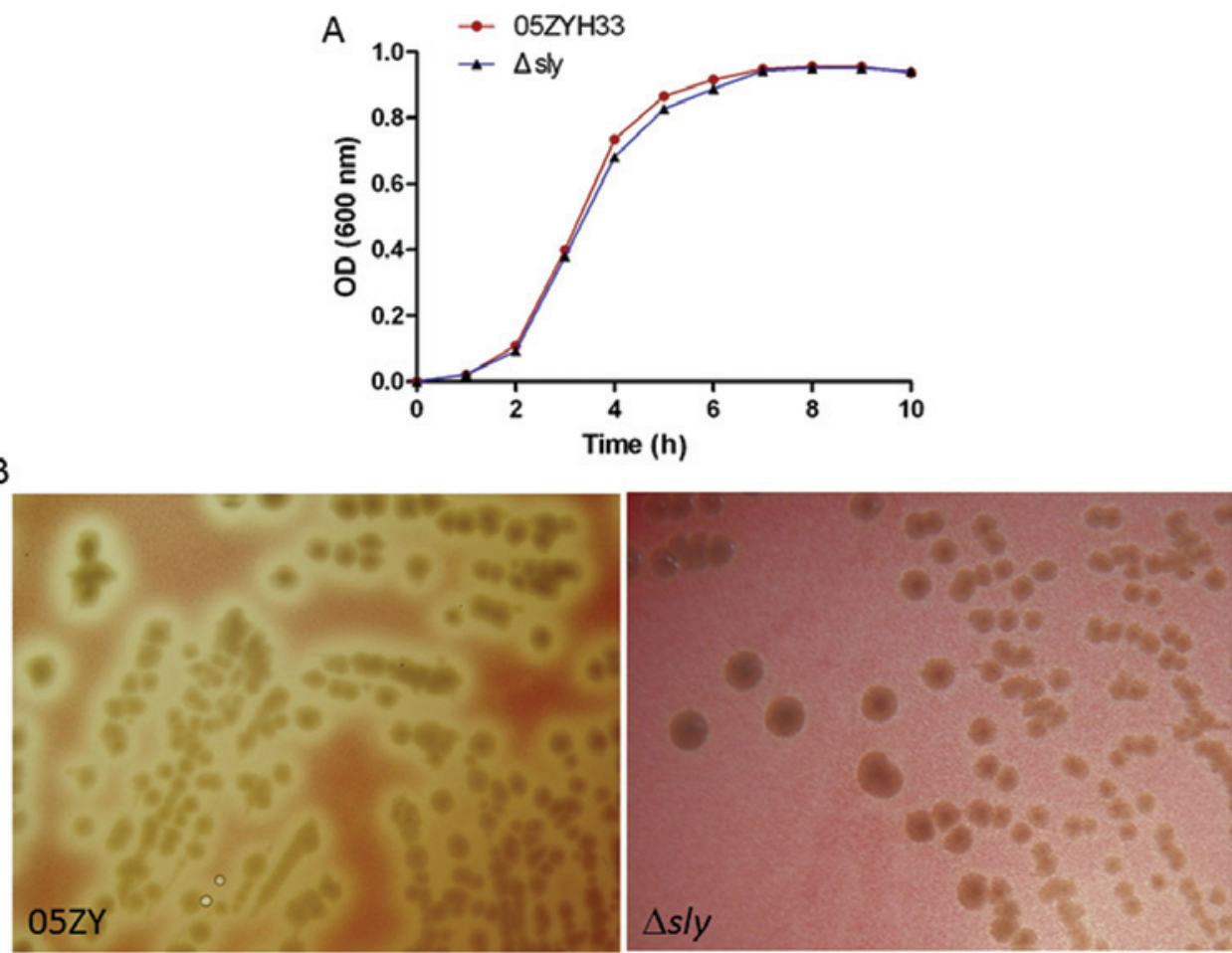

Figure 3. The mutant $\Delta s l y$ strain shows (A) similar growth rates compared to the wild-type strain S. suis 05ZYH33, but (B) reduced hemolytic activity.
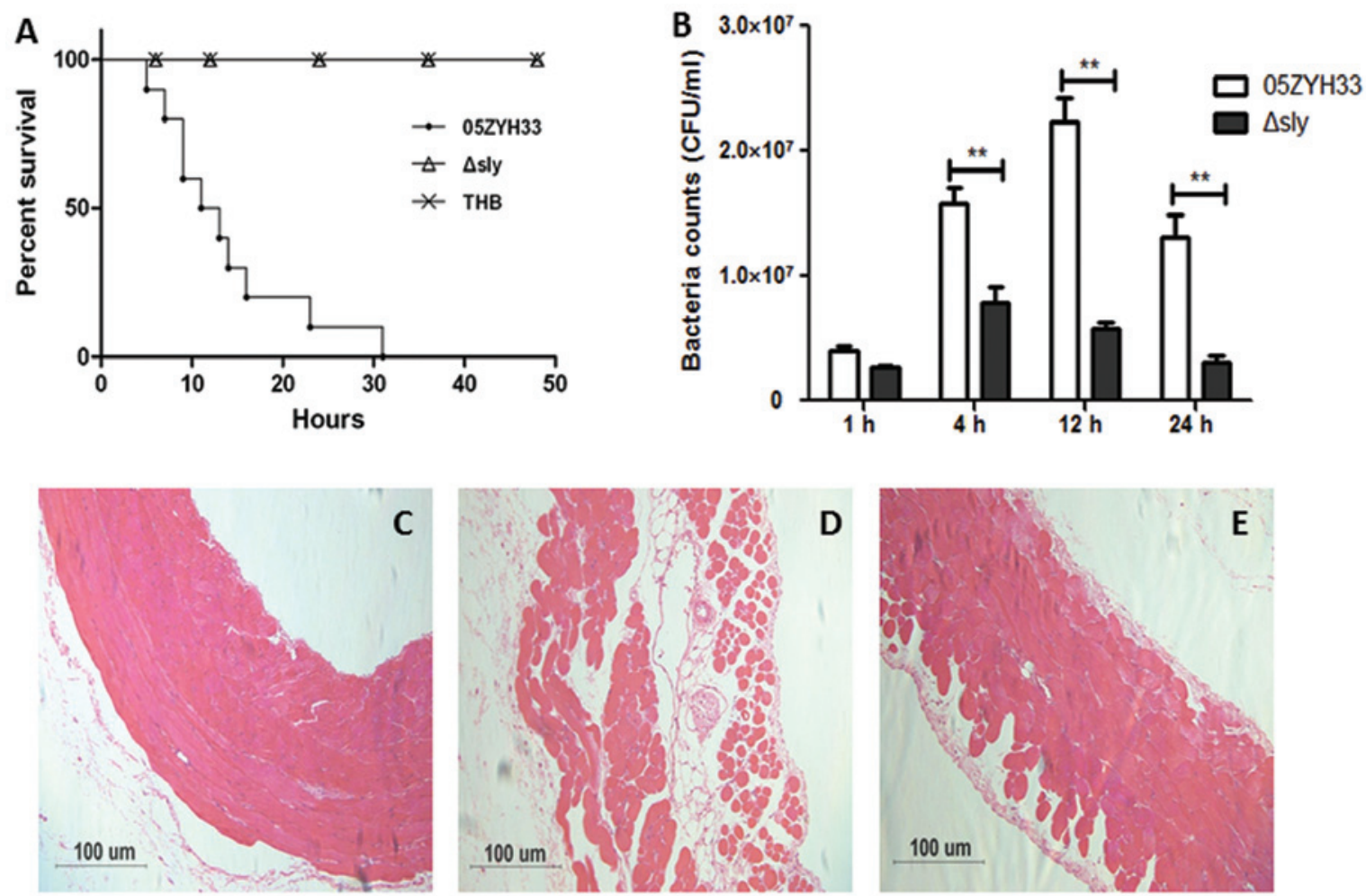

Figure 4. Suilysin is associated with the enhanced severity of S. suis 05ZYH33 infections. CD1 mice (6-week-old, female, $22 \mathrm{~g}$ ) were intraperitoneally injected with $0.5 \mathrm{ml}\left(\sim 5 \times 10^{8} \mathrm{CFUs}\right) S$. suis at the late-exponential growth phase, supplemented with Todd-Hewitt broth (THB). (A) The mortality of the infected mice, recorded for $50 \mathrm{~h}$. (B) The bacterial count in the blood at different time-points post-infection. The abdominal wall tissues were histopathologically examined $6 \mathrm{~h}$ after injection of (C) THB, (D) 05ZYH33, and (E) 4 sly, using hematoxylin and eosin staining. Images are representative of two independent experiments.

in sly expression between the two S. suis strains; a 6.3-fold difference at the mRNA transcription level of sly was observed between the two strains (data not shown).
In order to confirm the hypothesis that the S. suis ST7 epidemic strains may have increased production of sly compared to the non-epidemic strains, 11 S. suis ST7 epidemic 
strains and eight non-epidemic strains were collected (Table I), and the amount of sly was determined in the supernatant at $7 \mathrm{~h}$ of culture, using a sandwich ELISA assay (Fig. 2C). The epidemic ST7 strains produced more sly (mean, $1.49 \mathrm{~g} / \mathrm{ml}$; range, $0.76-1.91 \mathrm{~g} / \mathrm{ml}$ ) than the non-epidemic strains (mean, $0.33 \mathrm{~g} / \mathrm{ml}$; range, $0.07-0.94 \mathrm{~g} / \mathrm{ml}$ ). Moreover, the difference between the two groups was significant $(\mathrm{p}<0.001)$.

Sly is associated with enhanced severity of S. suis infections. To further explore the roles of sly in invasive infection of the 05ZYH33 strain, the sly gene was in-frame substituted by the $C m$ gene in the $S$. suis $05 Z$ YH33 strain, to generate the nonpolar mutant $\Delta s l y$. The mutant $\Delta$ sly strain showed reduced hemolytic activity, but similar growth rates compared to the wild-type strain S. suis 05ZYH33 (Fig. 3). We further investigated the virulence of this mutant. CD1 mice (6-week-old female, $\sim 22 \mathrm{~g}$ ) were infected by intraperitoneal injection with $0.5 \mathrm{ml}\left(\sim 5 \times 10^{8}\right.$ CFUs) $S$. suis at the late-exponential growth phase in THB. The mortality of the infected mice was recorded for $50 \mathrm{~h}$. No mice died in the $\Delta s l y$ or the THB control group, while the mice infected with $S$. suis $05 Z$ YH33 all died within $32 \mathrm{~h}$ post-infection. The bacterial concentration in the blood was evaluated at different time-points post-infection (Fig. 4A). The parental 05ZYH33 strain survived/multiplied in the blood at higher rates than $\Delta s l y$ at 4, 12 and $24 \mathrm{~h}$ (Fig. 4B). Histopathological examination of the abdominal wall tissues was also performed $6 \mathrm{~h}$ after the infection. The abdominal epithelial cells of mice infected by $S$. suis 05ZYH33 lost their normal morphological features and the integrity of the tight junctions, while the epithelial cells infected by $S$. suis $\Delta$ sly showed a few changes (Fig. 4C-E). Taken together, these data demonstrate that sly is associated with enhanced severity of S. suis 05ZYH33 infections.

\section{Discussion}

S. suis is a swine pathogen responsible for a number of infections, including meningitis, endocarditis and septicemiae, and is also an important zoonotic agent. The two outbreaks that occurred in China in 1998 and 2005 affected the world perspective regarding the threat that this pathogen represents for humans. Subsequent investigations on S. suis at the molecular and the genomic level confirmed that the emergence of the highly virulent SS2 strains, or epidemic ST7 strains, played a key role in these severe outbreaks (13). In the present study, we sought to determine the capacity of the S. suis protein sly to enhance the virulence of the epidemic ST7 strain 05ZYH33.

A previous study showed that $S$. suis ST7 strains express the proposed virulence markers muramidase-released protein (MRP), extracellular protein factor (EF), and sly (15). However to date, there is no study that specifically focused on how the differences in the expression abundance of bacterial virulence factors may affect the severity of S. suis infections. We hereby showed, using an animal model, that the $S$. suis ST7 epidemic strain 05ZYH33 causes higher mortality, higher necrosis of PMNs and significantly higher damage to HUVECs than the S. suis non-epidemic strain 1940. In agreement with these results, epidemic ST7 strains produced more sly than non-epidemic strains. This significant difference in sly secretion may be of outmost importance in S. suis
05ZYH33 infections. Phagocytosis is the first line of cellular defense against acute infectious diseases. A clinical association with invasive human infections implies that bacteria can survive the innate host defense responses raised in order to clear the bacteria from the bloodstream and the tissues. In other pathogenic streptococcal infections, hemolysins have been shown to be involved in bacteria-phagocyte interactions. For example, in Group B Streptococcus (GBS) infections, Liu et al (32) elegantly demonstrated the multi-factorial roles of the pore-forming toxin $\beta$-hemolysin/cytolysin $(\beta \mathrm{H} / \mathrm{C})$ in thwarting the immune phagocytic defenses. In the present study, we demonstrated that sly mediates inhibition of the phagocytic uptake by human PMNs in vitro. Moreover, we demonstrated that the $S$. suis strain with enhanced sly expression causes enhanced PMN necrosis in an in vivo mouse model. Taken together, these findings suggest that the sly-mediated inhibition of phagocytosis and cytolysis is directly associated with a mechanism impairing the function and viability of PMNs, and thus contributing to the enhanced survival of epidemic $S$. suis strains.

The ability of sly to enhance the severity of $S$. suis infection was confirmed using a mutant of $S$. suis $05 Z$ YH33 deficient in sly expression. The survival rate of mice challenged by the $\Delta s l y$ strain was significantly increased, and the bacterial count in the blood decreased compared to the parental strain 05ZYH33. These data strongly support the contribution of sly in enhancing the severity of S. suis ST7 strain infections, and are consistent with the previous study of Allen et al (33).

In our intraperitoneal infection animal model, $S$. suis needs to successfully cross the abdominal epithelial barrier and survive in the bloodstream in order to cause invasive infection. Among the potential strategies that can allow this are: transcellular transport by passive or adhesion-induced transcytosis, paracellular passage through opened tight junctions, disruption of the barrier due to a direct cytotoxic effect, leukocyte-facilitated transport by infected phagocytes (34). Histopathological examination showed that the mice infected by S. suis $05 Z$ YH33 lost their abdominal epithelial cell morphological features and the integrity of the tight junctions compared to mice infected by the mutant $\Delta s l y$. The $S$. pneumoniae toxin pneumolysin recognizes Toll-like receptor (TLR)4 on dendritic cells (35), and $S$. pneumoniae and $H$. influenzae were shown to exploit TLR-dependent downregulation of claudins 7 and 10, tight junction key components for the maintenance of the epithelial barrier integrity(36), to facilitate translocation across the epithelium (37). However, in vivo studies carried out in our laboratory indicate that the $S$. suis sly protein contributes to the release of inflammatory cytokines in a TLR4-independent manner (data not shown). Whether activation of TLR4 by sly can facilitate the $S$. suis translocation across the epithelium needs to be further studied.

In conclusion, we have shown that differences in sly production are linked to differences in $S$. suis virulence. The pathogenic $S$. suis ST7 strain 05ZYH33 appeared to have an increased ability to subvert host clearance mechanisms, which may allow its survival and dissemination into the bloodstream. The cytotoxic effects of sly may inhibit the uptake of microorganisms by the host phagocytes and can directly cause epithelial cell damage, enhancing the microbial spread into deeper tissues. Thus, in a murine model, sly contributes 
to increased bacterial load and death. Further understanding of the precise molecular mechanisms underlying the sly-mediated pathogenicity may provide potential therapeutic targets for a better control of $S$. suis infections.

\section{Acknowledgements}

This study was supported by grants from the Natural Sciences Foundation of China (30870091 and 81171528). We thank Professor Henk J. Wisselink and Marcelo Gottschalk for kindly providing S. suis strains.

\section{References}

1. Lun ZR, Wang QP, Chen XG, Li AX and Zhu XQ Streptococcus suis: an emerging zoonotic pathogen. Lancet Infect Dis 7: 201-209, 2007.

2. Segura M: Streptococcus suis: an emerging human threat. J Infect Dis 199: 4-6, 2009.

3. Wertheim HF, Nghia HD, Taylor W and Schultsz C: Streptococcus suis: an emerging human pathogen. Clin Infect Dis 48: 617-625, 2009.

4. Hui AC, Ng KC, Tong PY, Mok V, Chow KM, Wu A and Wong LK: Bacterial meningitis in Hong Kong: 10-years' experience. Clin Neurol Neurosurg 107: 366-370, 2005.

5. Mai NT, Hoa NT, Nga TV, et al: Streptococcus suis meningitis in adults in Vietnam. Clin Infect Dis 46: 659-667, 2008.

6. Yu H, Jing H, Chen Z, et al: Human Streptococcus suis outbreak, Sichuan, China. Emerg Infect Dis 12: 914-920, 2006.

7. Gottschalk M, Segura M, Xu J: Streptococcus suis infections in humans: the Chinese experience and the situation in North America. Anim Health Res Rev 8: 29-45, 2007.

8. Normile D: Infectious diseases. WHO probes deadliness of China's pig-borne disease. Science 309: 1308-1309, 2005.

9. Ngo TH, Tran TB, Tran TT, et al: Slaughterhouse pigs are a major reservoir of Streptococcus suis serotype 2 capable of causing human infection in southern Vietnam. PloS one 6 : e17943, 2011.

10. Chen C, Tang J, Dong W, et al: A glimpse of streptococcal toxic shock syndrome from comparative genomics of $S$. suis 2 Chinese isolates. PLoS One 2: e315, 2007.

11. Li M, Wang C, Feng Y, Pan X, et al: SalK/SalR, a two-component signal transduction system, is essential for full virulence of highly invasive Streptococcus suis serotype 2. PLoS One 3: e2080, 2008.

12. Li M, Shen X, Yan J, et al: GI-type T4SS-mediated horizontal transfer of the $89 \mathrm{~K}$ pathogenicity island in epidemic Streptococcus suis serotype 2. Mol Microbiol 79: 1670-1683, 2011.

13. Ye C, Zhu X, Jing H, et al: Streptococcus suis sequence type 7 outbreak, Sichuan, China. Emerg Infect Dis 12: 1203-1208, 2006.

14. Ye C, Bai X,Zhang J, et al: Spread of Streptococcus suis sequence type 7, China. Emerg Infect Dis 14: 787-791, 2008.

15. Ye C, Zheng H, Zhang J, et al: Clinical, experimental, and genomic differences between intermediately pathogenic, highly pathogenic, and epidemic Streptococcus suis. J Infect Dis 199: 97-107, 2009.

16. Alouf JE: Molecular features of the cytolytic pore-forming bacterial protein toxins. Folia Microbiol (Praha) 48: 5-16, 2003.

17. Segura M and Gottschalk M: Streptococcus suis interactions with the murine macrophage cell line J774: adhesion and cytotoxicity. Infect Immun 70: 4312-4322, 2002.

18. Lalonde M, Segura M, Lacouture S and Gottschalk M: Interactions between Streptococcus suis serotype 2 and different epithelial cell lines. Microbiology 146: 1913-1921, 2000 .
19. Charland N, Nizet V, Rubens CE, Kim KS, Lacouture S and Gottschalk M: Streptococcus suis serotype 2 interactions with human brain microvascular endothelial cells. Infect Immun 68: 637-643, 2000

20. Jacobs AA, Loeffen PL, van den Berg AJ and Storm PK: Identification, purification, and characterization of a thiolactivated hemolysin (suilysin) of Streptococcus suis. Infect Immun 62: 1742-1748, 1994.

21. Jacobs AA, van den Berg AJ and Loeffen PL: Protection of experimentally infected pigs by suilysin, the thiol-activated haemolysin of Streptococcus suis. Vet Rec 139: 225-228, 1996.

22. Pian Y, Gan S, Wang S, et al: Fhb, a novel factor H-binding surface protein, contributes to the antiphagocytic ability and virulence of Streptococcus suis. Infect Immun 80: 2402-2413, 2012.

23. Lv QY, Hao HJ, Bi LL, Zheng YL, Jiang YQ and Lv SX: Purification and biological activities analysis of streptococcus suis Serotype 2 suilysin. Xi Bao Yu Fen Zi Mian Yi Xue Za Zhi 27: 374-376, 2011 (In Chinese).

24. Gottschalk MG, Lacouture S and Dubreuil JD: Characterization of Streptococcus suis capsular type 2 haemolysin. Microbiology 141: 189-195, 1995.

25. Berthelot-Hérault F, Gottschalk M, Morvan H and Kobisch M: Dilemma of virulence of Streptococcus suis: Canadian isolate 89-1591 characterized as a virulent strain using a standardized experimental model in pigs. Can J Vet Res 69: 236-240, 2005

26. Wisselink HJ, Joosten JJ and Smith HE: Multiplex PCR assays for simultaneous detection of six major serotypes and two virulenceassociated phenotypes of Streptococcus suis in tonsillar specimens from pigs. J Clin Microbiol 40: 2922-2929, 2002.

27. Li Y, Martinez G, Gottschalk M, et al: Identification of a surface protein of Streptococcus suis and evaluation of its immunogenic and protective capacity in pigs. Infect Immun 74: 305-312, 2006.

28. Wisselink HJ, Reek FH, Vecht U, Stockhofe-Zurwieden N, Smits MA and Smith HE: Detection of virulent strains of Streptococcus suis type 2 and highly virulent strains of Streptococcus suis type 1 in tonsillar specimens of pigs by PCR. Vet Microbiol 67: 143-157, 1999.

29. Takamatsu D, Osaki M and Sekizaki T: Thermosensitive suicide vectors for gene replacement in Streptococcus suis. Plasmid 46: 140-148, 2001.

30. Schmittgen TD and Zakrajsek BA: Effect of experimental treatment on housekeeping gene expression: validation by real-time, quantitative RT-PCR. J Biochem Biophys Methods 46: 69-81, 2000.

31. Livak KJ and Schmittgen TD: Analysis of relative gene expression data using real-time quantitative PCR and the $2^{-\Delta \Delta C T}$ method. Methods 25: 402-408, 2001.

32. Liu GY, Doran KS, Lawrence T, Turkson N, Puliti M, Tissi L and Nizet V: Sword and shield: linked group B streptococcal beta-hemolysin/cytolysin and carotenoid pigment function to subvert host phagocyte defense. Proc Natl Acad Sci USA 101: 14491-14496, 2004.

33. Allen AG, Bolitho S, Lindsay $\mathrm{H}$, et al: Generation and characterization of a defined mutant of Streptococcus suis lacking suilysin. Infect Immun 69: 2732-2735, 2001.

34. Join-Lambert O, Morand PC, Carbonnelle E, Coureuil M, Bille E, Bourdoulous S and Nassif X: Mechanisms of meningeal invasion by a bacterial extracellular pathogen, the example of Neisseria meningitidis. Prog Neurobiol 91: 130-139, 2010.

35. Bernatoniene J, Zhang Q, Dogan S, Mitchell TJ, Paton JC and Finn A: Induction of CC and CXC chemokines in human antigen-presenting dendritic cells by the pneumococcal proteins pneumolysin and $\mathrm{CbpA}$, and the role played by toll-like receptor 4, NF-kappaB, and mitogen-activated protein kinases. J Infect Dis 198: 1823-1833, 2008.

36. Dominguez-Punaro MC, Segura M, Plante MM, Lacouture S, Rivest S and Gottschalk M: Streptococcus suis serotype 2, an important swine and human pathogen, induces strong systemic and cerebral inflammatory responses in a mouse model of infection. J Immunol 179: 1842-1854, 2007

37. Clarke TB, Francella N, Huegel A and Weiser JN: Invasive bacterial pathogens exploit TLR-mediated downregulation of tight junction components to facilitate translocation across the epithelium. Cell Host Microbe 9: 404-414, 2011. 